\title{
Factors Influencing Adolescent's Relationship With Non-Custodial Parents
}

\author{
Sung Hui Cho \\ Department of Social Welfare, HyupSung University, Hwaseong, Korea \\ 이혼가족 청소년의 비양육부모와의 관계에 관한 연구 \\ 조성희 \\ 협성대학교 사회복지학과
}

\begin{abstract}
Objective: This study examined the facotrs influencing the relationship of adolescents with noncustodial parent. Specifically, this study focused on the comparative influence of the family function as a factor controlling other factors such as socio-demographic characteristics, beliefs about parental divorce, and social support.

Methods: Data were collected from 322 adolescents from divorced families using a structured questionnaire. SPSS 22.0, descriptive statistical analysis, correlation analysis, and hierarchical multiple regression were performed to analyze the data.

Results and Conclusion: The results revealed that beliefs about parental divorce, social support, and family function affected the relationship with non-custodial parent. After controlling the influence of other factors, family function was found to have a significant influence on the relationship with noncustodial parents. Based on the results, practical suggestions were provided to enhance the relationship between adolescents and non-custodial parents.
\end{abstract}

Keywords: relationship with non-custodial parent, adolescent from divorced family, beliefs about parental divorce, social support, family function$$
\text { 서론 }
$$

우리 사회가 경험하는 다양한 변화 중 가장 대표적인 현상은 가족의 변화이다. 부부체계가 약화되면서 이혼으로 인한 가 족 해체가 심화되고 있으며, 이러한 가족 형태 변화 현상은 가 족주의의 약화로 인해 더욱 심화되고 있다(Y. S. Cho \& Lee, 2004; Moon, 2007). 인구 1,000명당 이혼건수를 의미하는 조 이혼율의 변화를 통해 이러한 변화를 살펴볼 수 있는데, 1990 년 1.1 건 정도였던 조이혼율은 1997 년 외환위기를 지나면서

Corresponding Author: Sung Hui Cho, Department of Welfare, Hyupsung University, 72, Choerubaek-Ro, Bongdam-Eup, Hwaseong-Si, GyeonggiDo, Korea, 18330.

E-mail: chosh@uhs.ac.kr
\end{abstract}

2.0건 정도로 급증하였으며, 이후 2005년 2.6건으로 지속적 인 증가현상을 보이다 현재는 2.3 건을 유지하고 있다(Statistics Korea, 2014). 조이혼율은 OECD 국가들과 비슷한 수준이지만 상대적으로 이혼가족에 대한 지원 체계가 부족하고, 사회적인 낙인으로 인해 발생하는 어려움이 큰 우리사회의 특성을 고려 하여 볼 때 이혼으로 이루어진 한부모가족이 경험하는 어려움 의 수준은 더 높다고 할 수 있다.

이혼으로 발생한 한부모가족이 경험하는 다양한 어려움 중 전통적으로 많이 제시된 것은 부모의 관점에서 한명의 부모

(C) The Korean Association of Child Studies

This is an Open Access article distributed under the terms of the Creative Commons Attribution Non-Commercial License (http:// creativecommons.org/licenses/by-nc/4.0) which permits unrestricted noncommercial use, distribution, and reproduction in any medium, provided the original work is properly cited. 
가 양부모의 역할을 모두 담당하면서 발생하게 되는 역할 과 중, 빈곤, 자녀 양육의 어려움 등이다. 하지만 부모의 이혼을 경험한 자녀들 역시 역할혼란, 부모 역할을 감당함으로 인한 가족 갈등, 자기 비난이나 불안 등과 같은 심리.정서적 문제, 대인 관계의 문제로 인한 어려움을 경험하게 된다(Amato \& Cheadle, 2005; E. J. Kim \& Baek, 2007).

하지만 가족 전체의 어려움을 가져오게 되는 이혼에 대 한 사회적 접근은 여전히 성인 부부를 중심으로 형성되어 있 다. 이혼숙려제도, 이혼전후상담 등의 제도 속에서 자녀에 대 한 개입의 정도는 낮은 수준이고, 이혼 이후 한부모 가족을 형 성하게 된 이후에도 빈곤의 문제에 초점을 맞춰서 개입이 이 루어지고 있다. 이로 인해 이혼과정과 그 이후 한부모 가족에 서 생활하는 이혼가족 청소년들에 대한 개입은 거의 이루어지 고 있지 않아 이들의 어려움은 개인적으로 해결해야 할 문제 로 남아 있다(Joo \& Cho, 2004). 실제적으로 한 세대에서의 이 혼은 가족체계 전체에 영향을 주게 되고, 자녀 세대의 어려움 을 증가시키게 된다(Amato \& Cheadle, 2005; Emery, 1999; K. E. Lee \& Ju, 2005). 이와 함께 이혼가족 자녀의 어려움은 부부가 이혼하는 시점에 절반 정도가 미성년 자녀를 두고 있다는 점 에서 더욱 주목하여 볼 필요가 있다(Statistics Korea, 2014).

상당수의 이혼가족에서 생활하고 있는 청소년은 다양한 측 면에서 어려움을 경험하게 되는데 특히 심리적인 측면과 사회 관계적인 측면에서 어려움을 경험하게 된다. 이러한 어려움 은 부모의 이혼이라는 사건 자체가 아니라 이혼과 관련된 현 상을 잘못 다루는 것으로 인해 발생하는 측면이 강해 자녀들 이 경험하는 어려움을 잘못 다룰 경우 내현화되거나 외현화된 형태의 문제를 발생시키게 된다(Emery, 2004). 즉 부모의 이 혼은 전통적 가족에 비해 가족기능의 제한을 가져올 수 있으 며, 이로 인하여 자녀들은 다양한 심리적·사회적인 문제들에 노출될 높은 가능성을 경험하게 된다(Y. S. Cho \& Lee, 2004; Felner, 2006; Masten \& Coastsworth, 1998; Wallerstein, Kelly, \& Blakelee, 2000). 이혼가족의 자녀들은 비이혼가족의 자녀들 에 비해 생활 속에서 상대적으로 높은 수준의 불안과 스트레 스를 경험하게 되고, 비행과 문제 행동의 발생 가능성이 높으 며, 이와 관련하여 정신과 상담 서비스를 받게 되는 경우가 높 게 관찰된다(Emery, 1999; Joo \& Cho, 2004; Wallerstein, Kelly, $\&$ Blakelee, 2000). 또한 부모의 이혼을 경험한 청소년들의 경 우에는 자아존중감의 저하와 인식능력의 결함을 포함하는 인 지적인 어려움을 경험하는 비율 역시 높게 관찰되고 있다. 아 동기에서 성인기로 전환하는 과정이며, 신체적·심리적·사회적 적응을 위하여 다양한 차원에서의 혼란과 불안을 경험하는 청
소년기의 상황과 맞물리면서 이는 더 큰 위험을 가져오게 된 다(Hong, 2004; Y. Y. Park \& Kim, 2005).

부모의 이혼으로 경험하게 되는 다양한 어려움과 위험 요 인들 속에서 이혼가족 청소년들을 보호하고 온전하게 성장하 도록 지원하는 다양한 보호요인들도 제시되고 있다. 그런데 부모의 이혼이라는 사건으로 인해 발생한 가족체계의 변화로 인해 어려움을 경험한 청소년들이 복지감을 획득하고, 성인으 로 온전히 성장하는데 영향을 주는 가장 큰 요인은 다시 가족 으로 귀결되게 된다(Falci, 2006; Risch, Jodl, \& Eccles, 2004). 하 지만 이혼 청소년에게는 가족이 하나가 아니며, 실제적으로 두 개의 가족이 존재하게 되어 두개의 가족과의 모든 상호작 용은 청소년이 온전한 성장을 하는 중요한 요인으로 작용하게 된다. 즉 함께 생활하지 않지만 부모로서 역할을 수행하고 있 고, 수행해야 할 필요성을 가진 비양육 부모가 생긴다(Depner \& Bray, 1993). 이로 인해 이혼가족 청소년들을 지원하고 보 호하는 가족을 고려할 때 현재 동거하는 가족과의 상호작용 과 함께 동거하지는 않지만 실제적으로 밀접한 상호작용을 하 게 되는 비양육부모와의 관계를 함께 고려하는 것은 매우 중 요하다(Amato, 2000; Choi, 2013; Chung, 1993; Depner \& Bray, 1993; S. Y. Lee, 2002; Son, 2013). 비양육부모와의 관계는 이혼 가족 청소년이 경험하는 어려움을 완화하고, 이들이 사회적으 로 기능하는데 기여할 수 있는 중요한 요인으로 작용하게 된 다(Son, 2013).

이혼가족 청소년의 비양육부모와의 관계를 검토함에 있어 서 청소년들의 성장에 영향을 주는 다양한 체계가 가지는 특 성과 이들에 대한 종합적인 검토가 필요하다. 먼저 비양육부 모와의 관계에 영향을 주는 요인으로 살펴볼 수 있는 것은 부 모의 이혼 경험으로 인해 청소년에게 발생하는 부모이혼에 대 한 지각이다. 부모이혼지각이란 청소년이 부모의 이혼을 경험 하면서 갖게 되는 신념으로 이혼으로 인해 변화된 상황에 대 한 지각을 포함하는 개념이다(Kurdek \& Berg, 1987). 이혼에 대한 부정적인 지각은 이혼가족 청소년의 부모와의 관계뿐만 아니라 사회적인 상호작용의 감소와 일탈에 영향을 주는 것으 로 알려져 있다(Haggerty, Sherrod, Garmezy, \& Rutter, 1996; E. S. Kang \& Yi, 2013). 특히 부부의 갈등이 심한 상황에서 이혼 이 발생하는 경우 무기력, 분노, 비난을 포함하는 부모이혼에 대한 문제성 인식이 증가해 비양육부모와의 관계 형성에 부정 적인 영향을 주는 것으로 제시되고 있다(Joo \& Cho, 2004; Ju, 2003; Kurdek, 2002).

또한 이혼가족 청소년과 비양육부모와의 관계를 탐색함에 있어서 학교를 중심으로 한 또래관계와 교사와의 관계에 영향 
을 많이 받게 되는 청소년들의 특성을 반영하여 사회적 상호 작용에 대한 고려도 중요하다(Choi, 2013; Hetherington, 2003; Y. Y. Park \& Kim, 2005). 청소년의 사회적 상호작용은 청소년 에게 가장 주요하게 영향을 주는 체계인 학교와 실질적인 상 호작용을 하게 되는 교사와 또래의 사회적지지를 통해 살펴볼 수 있다. 이혼가족 청소년들이 경험하는 사회적지지는 이들의 문제 행동, 적응 능력에 영향을 주게 되며(Demaray, Malecki, Davidson, Hodgson, \& Rebus, 2005), 안정적인 사회적지지 자 원이 많은 학교 환경을 가지고 있는 경우 비양육부모와의 관 계 및 안정적인 적응에 긍정적인 도움을 주는 것으로 제시되 었다(Jang \& Lee, 2011; Lee \& Joo, 2005).

마지막으로 이혼가족 청소년이 경험하는 비양육부모와의 관계를 살펴봄에 있어서 이들에게 가장 중요한 영향을 주는 현재 함께 생활하는 가족과의 상호작용을 살펴보아야 한다. 현재 함께 생활하는 가족과의 상호작용을 의미하는 가족기능 은 가족구성원들간의 정서적 유대감과 결속력을 통해 스트레 스를 기능적으로 해결하는 능력을 의미한다(Epstein, Baldwin, $\&$ Bishop, 1983). 이러한 가족기능은 부모의 이혼을 경험한 청 소년들이 변화된 상황에 적응하고, 온전한 성장을 가져오게 하는 가장 중요한 요소로 제시되고 있다(Choi, 2013; Ju, 2003; Son, 2013). 현재 함께 생활하는 가족으로부터의 가족기능이 원활하지 않고 왜곡된 형태의 상호작용이 이루어지는 경우 다 양한 문제 행동이 발생하며, 비양육부모와의 관계를 포함해 변화된 상황에 대한 적응력이 낮아지는 것으로 나타나고 있다 (S. Y. Lee, 2002).

또한 이혼가족 청소년의 비양육부모와의 관계에 영향을 주는 인구사회학적 특성으로는 성별, 연령, 경제수준, 형제유 무, 종교유무를 살펴볼 수 있다. 일반적으로 성별, 연령, 경제 수준에서는 여자 청소년의 경우, 연령이 적을수록, 부모의 경 제적 지원의 수준이 높을수록 부모와 긍정적인 관계를 형성 하는 것으로 나타나 있다(K. W. Kim \& Moon, 2005). 종교로 인해 형성되어지는 종교적 안녕 역시 부모-자녀간의 관계에 긍정적인 영향력을 갖는 것으로 나타나고 있으며(Carothers, Borkowski, Lefevre, \& Whitman, 2005; J. A. Park \& Yoo, 2003), 형제가 있는 경우가 그렇지 않은 경우에 비해 부모와의 관계 에 더 큰 애착을 갖고 있다는 것이 제시되어 왔다(Bae \& Lee, 2006).

그런데 우리나라의 이혼율과 이혼 당시 미성년 자녀의 비 율이 높고, 많은 어려움을 경험하는 이혼가족 청소년에게 비 양육부모와의 관계가 중요함에도 불구하고 관련된 연구는 매 우 부족한 실정이다(Y. K. Kim \& Han, 2006; Son, 2013). 지금
까지의 제한적으로 이루어진 이혼가족 청소년에 대한 연구는 크게 세 가지로 구분하여 볼 수 있는데, 첫 번째 영역은 이혼가 족 청소년의 정신건강이나 심리사회적 적응에 대한 연구이다 (H. S. Kim, 2005; Yoon, Lee, Kim, \& Jeong, 2012). 두 번째 영역 은 이혼 가족 청소년을 대상으로 한 상담이나 심리치료의 효 과성에 대한 연구이다(H. K. Kim \& Chang, 2015; Y. K. Kim \& Lee, 2011; S. Y. Lee, 2009). 세번째 영역은 미성년 자녀를 둔 이 혼가족을 대상으로 양육을 포함하여 부모 역할에 관한 연구이 다(Jun, 2002; Y. K. Kim \& Han, 2006; Yoo, 2005).

이혼가족 청소년을 대상으로 한 연구 중 국외에서 이루어 진 연구들(Haggerty et al., 1996; Hetherington, 2003; Kurdek, 2002)은 이혼 이후의 자녀 양육문제에 충분히 숙고할 수 있는 기간을 법제화하고, 이 과정 속에서 자녀 양육에 대한 합의와 공동부모로써의 역할이 조정되어질 수 있는 제도적인 장치가 마련된 환경에서 이루어졌다는 점에서 국내의 상황과 차이를 보이고 있다(S. J. Kim, 2008). 즉 이혼을 둘러싼 맥락 및 이혼 이후 비양육부모와의 상호작용 특성이 다르다는 점에서 국내 에 적용하는데 한계를 갖는 것이다. 이를 극복하기 위해 국내 에서 진행된 연구는 비양육부모와의 관계와 관련된 현상을 매 우 제한적으로 다루고 있다. 관련 연구들(Choi, 2013; Ju, 2003; Jun, 2002; Yoo, 2005)의 경우에는 비양육부모와의 관계에 대 해 제한적 사례를 대상으로 인터뷰를 통해 수집된 자료를 중 심으로 잠정적 결론을 도출하는 수준의 탐색적 연구들이 질적 인 연구 방법에 의해 수행되었다. 이로 인해 이혼가족 청소년 과 비양육부모와의 관계에 영향을 주는 변인들을 객관적으로 검증하고, 관련 현상을 이해하는데 제한을 갖고 있다. 연구에 서 검토되어진 변수에 있어서도 청소년에게 영향을 주는 개인 적인 측면, 학교체계에서의 상호작용, 동거가족과의 상호작용 을 종합적으로 살펴본 연구 역시 거의 이루어지지 않은 상태 이다. 또한 이혼가족 청소년들에게 가장 중요한 영향을 주는 현재 함께 생활하는 가족과의 상호작용이 비양육부모와의 관 계에 대해 갖는 독자적인 설명력과 특성을 검토한 연구는 수 행되지 않은 상황이다. 이에 본 연구에서는 이혼가족 청소년 들의 비양육 부모와의 관계에 영향을 주는 요인들을 종합적으 로 살펴보고, 이 과정 속에서 현재 함께 생활하는 가족과의 상 호작용이 갖는 독자적인 설명력을 검증함으로써 비양육부모 와의 관계에 있어 현재 함께 생활하는 가족과의 상호작용이 갖는 중요성을 살펴보고자 한다. 이를 기반으로 하여 이혼가 족 청소년과 비양육부모의 관계를 증진시키기 위한 방안을 모 색하고자 한다. 


\section{연구문제 1}

이혼가족 청소년의 부모이혼지각, 사회적지지, 가족기능, 비 양육부모와의 관계의 특성과 주요 특성간의 관계는 어떠한 가?

\section{연구문제 2}

인구사회학적 특성, 부모이혼지각, 사회적지지가 비양육부모 와의 관계에 미치는 영향과 다른 요인들을 통제한 상태에서 가족기능은 독자적인 설명력을 갖는가?

\section{연구방법}

\section{연구대상}

이혼가족 청소년들이 경험하는 비양육부모와의 관계에 영 향을 미치는 요인을 살펴보기 위하여 구조화된 설문지를 통 해 부모의 이혼을 경험한 이혼가족의 자녀 중 한명의 부모와 동거하고 있는 13 세에서 19 세의 청소년들을 대상으로 연구를 수행하였다. 연구대상자의 선정은 청소년을 대상으로 서비스 를 제공하고 있는 청소년상담기관, 중·고등학교, 사회복지기 관을 통해 이루어졌다. 최종적인 연구대상자는 연구의 목적과 자료 수집의 내용, 자료 활용 방법, 보호조치, 연구 거부 및 중 단 가능에 대한 설명 후 연구에 동의하는 과정을 통해 선발되 었다. 이를 통해 수집된 총 340 부의 조사자료 중 연구의 주요 변수에 불성실한 응답을 한 경우와 부정확한 응답이 이루어진 18 사례를 제외하고 최종 분석에는 322 명의 자료가 활용되었 다.

연구대상 이혼가족 청소년 322 명의 인구사회학적 특성은 Table 1과 같다. 성별에 있어 여학생인 경우가 167 명(51.9\%)으 로 남학생인 경우 155 명(48.1\%)에 비해 상대적으로 높은 비 율을 보였다. 연령은 14-16세인 경우가 169명(52.4\%)으로 가 장 많았고, 평균 16.49 세 $(S D=1.78)$ 로 나타났다. 형제는 있 는 경우가 256명(79.5\%)으로 형제가 없는 경우 66명(20.5\%) 에 비해 높은 비율을 차지하고 있었다. 청소년들이 인식하고 있는 가족의 경제적 수준에서는 보통인 4점인 경우가 125 명 (38.8\%), 3점인 경우가 99명(30.1\%)의 순으로 높은 비율을 보 였으며, 평균 3.31 $(S D=1.10)$ 로 보통보다 낮은 수준에서 가 족의 경제 수준을 인식하고 있었다. 종교적 특성에서는 종교 가 있는 경우가 191명(59.3\%)으로 종교가 없는 경우 131명 (40.7\%)에 비해 높은 비율을 보였다. 부모의 이혼으로 인해 현
Table 1

Participants' Characteristics

\begin{tabular}{|c|c|c|}
\hline Categorie & $N$ & $\%$ \\
\hline \multicolumn{3}{|l|}{ Gender } \\
\hline $\mathrm{M}$ & 155 & 48.1 \\
\hline $\mathrm{F}$ & 167 & 51.9 \\
\hline
\end{tabular}

Age

$\begin{array}{lll}13 & 4 & 1.2\end{array}$

$\begin{array}{lll}14-16 & 169 & 52.4\end{array}$

$\begin{array}{lll}\geq 17 & 149 & 46.4\end{array}$

$M(S D) \quad=16.49(1.78)$

Siblings

$\begin{array}{lll}\text { Yes } & 256 & 79.5\end{array}$

$\begin{array}{lll}\text { No } & 66 & 20.5\end{array}$

Economic status

$\begin{array}{lrr}1 \text { (Very low) } & 17 & 5.3 \\ 2 & 57 & 17.7 \\ 3 & 99 & 30.1 \\ 4 \text { (Middle) } & 125 & 38.8 \\ 5 & 17 & 5.3 \\ 6 & 6 & 1.9 \\ 7 \text { (Vert high) } & 3 & 0.9 \\ M(S D) & & 3.31(1.10)\end{array}$

Religion

$\begin{array}{lll}\text { Yes } & 191 & 59.3\end{array}$

$\begin{array}{lll}\text { No } & 131 & 40.7\end{array}$

Period of single-parent family formation (years)

$\begin{array}{lll}<2 & 34 & 10.6\end{array}$

$2 \leq$ years $<4 \quad 59 \quad 18.3$

$4 \leq$ years $<6 \quad 51 \quad 15.8$

$6 \leq$ years $<8 \quad 71 \quad 25.2$

$8 \leq$ years $<10 \quad 36 \quad 11.2$

$\begin{array}{lll}>10 & 61 & 18.9\end{array}$

$M(S D) \quad 6.18(3.90)$

Note. $N=322$.

재의 한부모와 생활한 기간은 6년 이상 8년 미만인 경우가 71 명(25.2\%), 10 년 이상인 경우가 61명(18.9\%), 2년 이상 4년 미 만인 경우가 59명(18.3\%)의 순으로 높은 비율을 보였고, 평균 6.18년 $(S D=3.90)$ 으로 나타났다. 


\section{연구도구}

\section{비양육부모와의 관계}

비양육부모와의 관계는 학습이론과 체계이론에 기반하여 Lange 등(1998)이 개발한 Parent-Child Interaction Questionnaire (PACHIQ)를 기초로 하여 Lange 등(2002)이 수정 보완한 Parent-Child Interaction Questionnaire-Revised (PACHIQ-R) 중 자녀용 측정도구(PACHIQ-R-CH)를 통해 측정하였다. 이 도 구는 총 25 문항을 통해 부모와 자녀간의 상호작용을 통해 형 성되어지는 관계를 측정하고 있으며, 하위요인으로 갈등해 결(conflict resolution)과 수용(acceptance)이라는 두 가지 요인 을 제시하고 있다. 갈등해결에는 "어머니(아버지)와 나 사이 에 가끔 해결할 수 없는 문제가 있다”와 같은 문항이 포함되어 있으며, 수용에는 "내가 슬플 때 어머니(아버지)는 나를 위로 해 주신다"와 같은 문항을 포함하고 있다. 응답의 범주는 전혀 그렇지 않다(1)에서 항상 그렇다(5)의 5점 리커트 척도로 구성 되어 있으며, 점수가 높을수록 비양육부모와의 관계가 긍정적 임을 의미한다. Lange 등(2002)의 연구에서 PACHIQ-R-CH의 내적일치도(Cronbach's $\alpha$ )는 .90이었고, 본 연구의 내적일치도 (Cronbach's $\alpha$ )는 .88로 나타났다.

\section{부모이혼지각}

부모의 이혼과 관련해 나타나게 되는 특성 중 이혼가족 청소 년들이 부모의 이혼을 경험하면서 부모의 이혼에 대해 갖게 되는 지각을 살펴보기 위하여 Kurdek과 Berg (1987)가 개발한 Children's Beliefs About Parental Divorce Scale (CBAPDS)을 활 용하였다. 이 도구는 총 36문항 구성되어 부모의 이혼을 경험 한 자녀에게 나타날 수 있는 문제성 신념의 정도를 측정하게 된다. 또래놀림(peer ridicule and avoidance), 아버지에 대한 비 난(paternal blame), 어머니에 대한 비난(maternal blame), 유기 불안(ear of abandonment), 재결합환상(hope of reunification), 자 기 비난(self-blame)의 총 여섯 가지 하위요인으로 구성되며, 각 요인별로 6문항씩이 포함되어 있다. 또래놀림의 대표적인 문 항은 “다른 친구들이 부모님에 관해 많은 질문을 하면 화가 날 것 같다”이며, 아버지에 대한 비난과 어머니에 대한 비난은 각 각 “아버지가 우리 가족문제의 원인 제공자다", “어머니가 우 리 가족문제의 원인 제공자다"와 같은 문항을 포함하고 있다. 유기불안은 “나는 종종 내가 혼자 남겨질까 걱정이다"와 같은 문항을 포함하고 있으며, 재결합환상과 자기비난은 각각 “나
는 부모님이 언젠가 함께 살 것이라고 생각한다", "내가 말하 고 행동하는 것 때문에 부모님이 서로 불행해 한다"와 같은 문 항을 통해 측정되어진다. 응답의 범주는 예(1)와 아니오(2)로 구성되어 있으며, 응답의 점수가 높을수록 부모이혼 지각에 대한 문제성 신념이 낮음을 의미한다. Kurdek과 Berg (1987)의 연구에서 CBAPDS의 내적일치도(Cronbach's $\alpha$ )는 .80이었고, 본 연구에서의 내적일치도(Cronbach's $\alpha$ )는 .72로 나타났다.

\section{사회적지지}

연구대상인 이혼가족 청소년들이 경험하고 있는 사회적 상 호작용의 측정은 Malecki 등(1999)에 의해 제안된 Child and Adolescent Social Support Scale (CASSS)의 하위척도 중 교사지 지(teacher support)와 또래지지(peer support)를 통해 이루어졌 다. 교사지지는 “선생님은 나는 이해해 주신다"와 같은 문항 을 포함하고 있으며, 또래지지는 "친구들은 나의 감정을 이 해해 준다"의 문항을 통해 측정되어진다. 교사지지와 또래 지지는 각각 10 문항으로 각 문항의 응답은 전혀 그렇지 않다 (1)에서 항상 그렇다(6)의 6점 리처트 척도로 구성되어 있다. Malecki 등(1999)의 연구에서 CASSS의 내적일치도(Cronbach's $\alpha$ )는 .80으로 나타났고, 본 연구에서의 내적일치도(Cronbach's $\alpha)$ 는 .92로 나타났다.

$$
\text { 가족기능 }
$$

현재 이혼가족 청소년이 동거하고 있는 가족들과의 상호작용 을 의미하는 가족기능을 측정하기 위해 Epstein 등(1983)에 의 해 개발된 Family Assessment Device (FAD) 일곱 개의 하위요인 중 일반적 가족기능 (general functioning)을 활용하였다. FAD 의 하위요인들은 개별적으로 활용이 가능하며 일반적 가족기 능은 총 12 문항으로 구성되어 있다. 일반적 가족기능은 대표 적으로 "우리 가족은 문제를 해결하기 위한 의사결정을 잘 한 다"와 같은 문항을 포함하고 있다. 응답의 범주는 전혀 그렇지 않다(1)에서 매우 그렇다(4)의 4점 리커트 척도로 구성되어 있 으며, 점수가 높을수록 현재 동거하고 있는 가족들이 기능적 인 상호작용을 하고 있음을 의미한다. Epstein 등(1983)의 연구 에서 $\mathrm{FAD}$ 의 내적일치도(Cronbach's $\alpha$ )는 .92로 나타났고, 본 연구에서의 내적일치도(Cronbach's $\alpha$ )는 .91이었다. 


\section{인구사회학적 특성}

인구사회학적 특성은 성별, 연령, 형제유무, 경제수준, 종교유 무, 한부모가족 형성 기간을 중심으로 살펴보았다. 성별은 남 자(0), 여자(1)로 구분하여 활용하였고, 연령은 응답자들이 응 답한 만나이를 활용하였다. 형제유무는 형제가 있는 경우(0), 형제가 없는 경우(1)로 구분하였고, 경제수준은 연구대상자들 이 인지하고 있는 가족의 경제상태로 매우 나쁘다(1)에서 매우 좋음(7)의 7점 리커트 형태로 구성하여 측정하였다. 종교유무 는 종교유형에 상관없이 종교가 있는 경우(0), 종교가 없는 경 우(1)로 구분하였다. 한부모 가족 형성 기간은 부모의 이혼으 로 인해 현재의 한부모가족을 형성하게 된 기간을 측정하였다.

\section{자료분석}

이혼가족 청소년들이 인지하고 있는 비양육부모와의 관계에 영향을 주는 요인을 살펴보기 위해 본 연구에서는 기술통계분 석, 상관관계분석, 위계적 다중회귀분석(hierarchical multiple regression analysis)을 실시하였다. 영향요인에 대한 분석에 앞 서 이혼가족 청소년들의 인구사회학적 특성과 이혼가족청소 년들의 부모이혼지각, 사회적지지, 가족기능, 비양육부모와의 관계의 특성을 빈도분석과 기술통계분석을 통해 살펴보았다. 위계적 회귀분석의 전제조건인 독립변수들간의 다중공선성 의 문제 확인은 비양육부모와의 관계와 밀접한 관련성을 갖는 주요 변인들의 상관관계 분석을 통해 확인하였다. 최종적으 로 비양육부모와의 관계에 주요하게 관련되어 있는 영향 요인 을 인구사회학적 특성, 부모이혼지각, 사회적지지, 가족기능 의 순으로 순차적으로 모형에 투입하여 각 연구모형들의 변화
를 살펴볼 수 있는 위계적 다중회귀분석 통해 비양육부모와의 관계에 대한 주요변수들의 영향력과 다른 영향요인들을 통제 한 상태에서의 가족기능의 독자적인 설명력을 살펴보았다. 연 구문제를 해결에 있어서 필요한 통계적인 분석에는 SPSS 22.0 (IBM Co., Armonk, NY)이 활용되었다.

\section{연구결과}

\section{비양육부모와의 관계 및 주요 변수들의 특성 과 관련성}

이혼가족 청소년의 비양육부모와의 관계 및 주요변수들의 특 성을 살펴본 결과는 Table 2 와 같다. 주요 변수 중 부모의 이혼 지각의 경우 평균 $1.67(S D=.14)$ 로 응답범주를 고려하여 볼 때 중간보다 약간 높은 수준을 나타냈으며, 이는 이혼가족 청 소년들의 부모이혼에 대한 지각의 문제성 신념이 보통보다 낮 은 것을 의미한다. 현재 동거하고 있는 가족과의 상호작용을 의미하는 가족기능과 교사나 또래로부터 받고 있는 사회적지 지의 수준은 각각 $2.69(S D=.57)$ 와 $4.02(S D=.76)$ 로 나타났 고, 이는 가족기능과 사회적지지의 수준이 보통보다 약간 높 은 수준임을 나타낸다.

연구대상 청소년들이 응답한 비양육 부모와의 관계는 평균 $3.17(S D=.59)$ 로 보통보다 약간 높은 수준을 나타냈다. 이는 비양육부모의와 관계에 대해 보통보다 약간 높은 수준으로 긍 정적인 인식을 하고 있음을 의미한다. 연구의 주요 변수인 부 모이혼지각 $(r=.402, p<.001)$, 가족기능 $(r=.710, p<.001)$, 사 회적지지 $(r=.367, p<.001)$ 는 모두 비양육부모와의 관계에 정

Table 2

Variables' Characteristics and Correlation

\begin{tabular}{llll}
\hline \multicolumn{1}{c}{ Categories } & 1 & 2 & 3 \\
\hline $\begin{array}{l}\text { 1. Beliefs about parental } \\
\quad \text { divorce }\end{array}$ & - & & \\
2. Family function & $.380^{* * *}$ & - & \\
3. Social support & $.263^{* * *}$ & $.325^{* * *}$ & - \\
4. Relationship with non- & $.402^{* * *}$ & $.701^{* * *}$ & $.367^{* * *}$ \\
$\quad$ custodial parent & & & 4.02 \\
$M$ & 1.67 & 2.69 & .76 \\
$S D$ & .14 & .57 & 3.17 \\
\hline
\end{tabular}

Note. $N=322$.

${ }^{* * *} p<.001$. 
적으로 유의미한 상관관계를 갖고 있었다.

\section{비양육부모와의 관계의 영향 요인과 가족기능 의 독자적인 설명력}

이혼가족 청소년들이 비양육부모와 맺고 있는 관계에 영향을 주는 요인을 알아보기 위해 위계적 다중회귀분석을 실시하였 다. 회귀분석을 실시하기에 앞서 연구모형의 왜곡을 발생시킬 수 있는 다중공선성의 문제를 확인하기 위해 변수간 상관관 계, 공차한계(tolerance), 분산팽창요인(VIF)을 확인하였다. 앞 서 상관관계분석 결과에서 제시한 바와 같이 독립변수간 상관 관계가 가장 큰 것은 부모이혼지각과 가족기능으로 나타났다 $(r=.380, p<.001)$. 전체 연구 모형에서 공차한계에서는 가장 낮은 값이 .736, 분산팽창요인에서는 가장 높은 값이 1.358 로
나타났고, 이를 종합적으로 판단하여 볼 때 다중공선성의 문 제로 인해 연구모형이 왜곡될 가능성은 낮다고 판단하였다.

이혼가족 청소년의 비양육부모와의 관계에 영향을 주는 요 인을 살펴보기 위하여 인구사회학적 특성, 부모이혼지각, 사 회적지지, 가족기능을 위계적 회귀분석을 통해 순차적으로 투 입하였다. 새로운 변수가 투입되는 각 단계마다 모형 전체의 설명력과 추가적인 설명력 변화의 유의미성, 투입된 변수의 유의미성과 영향력을 Table 3에 제시하였으며, 이를 통해 비양 육부모와의 관계에 영향을 주는 요인을 제시하였다. 각 단계 의 모형이 갖는 특성 중 본 연구에서 살펴보고자 한 비양육부 모와의 관계에 대한 주요변수들의 영향력과 다른 요인들을 통 제한 상태에서 가족기능의 설명력을 살펴볼 수 있는 마지막 단계를 중심으로 살펴보았다.

3 단계 모형에 가족기능을 추가한 4단계 모형은 전체로 통계

Table 3

Multiple Regression Analysis of Relationship with Non-Custodial Parent

\begin{tabular}{|c|c|c|c|c|c|c|c|c|c|c|c|c|}
\hline \multirow[b]{2}{*}{ Variables } & \multicolumn{3}{|c|}{$1^{\text {st }}$ Model } & \multicolumn{3}{|c|}{$2^{\text {nd }}$ Model } & \multicolumn{3}{|c|}{$3^{\text {rd }}$ Model } & \multicolumn{3}{|c|}{$4^{\text {th }}$ Model } \\
\hline & B & $\beta$ & $t$ & B & $\beta$ & $t$ & $B$ & $\beta$ & $t$ & B & $\beta$ & $t$ \\
\hline Constant & 3.183 & & $9.143^{* * *}$ & .364 & & .734 & .051 & & .105 & -.261 & & -.689 \\
\hline Gender & -.044 & -.038 & -.677 & -.046 & -.039 & -.771 & -.039 & -.033 & -.682 & .031 & .026 & .667 \\
\hline Age & .017 & -.050 & -.901 & -.010 & -.031 & -.607 & -.010 & -.029 & -.588 & .022 & .068 & 1.697 \\
\hline Economic status & .092 & .171 & $3.050^{* *}$ & .079 & .146 & $2.828^{* *}$ & .070 & .129 & $2.598^{* *}$ & .022 & .041 & 1.019 \\
\hline Siblings & -.221 & -.151 & $-2.696^{* *}$ & -.176 & -.121 & $-2.327^{*}$ & -.152 & -.104 & $-2.089^{*}$ & -.106 & -.073 & -.1840 \\
\hline Religion & .014 & .012 & .214 & .003 & .002 & .049 & .032 & .027 & .545 & -.106 & -.073 & -1.840 \\
\hline $\begin{array}{l}\text { Period of single-parent } \\
\text { family formation }\end{array}$ & .002 & .016 & .288 & .002 & .014 & .266 & .000 & -.003 & -.062 & -.008 & -.050 & -1.273 \\
\hline $\begin{array}{l}\text { Beliefs about parental } \\
\text { divorce }\end{array}$ & & & & 1.654 & .381 & $7.484^{* * *}$ & 1.357 & .312 & $6.154^{* * *}$ & .556 & .128 & $3.041^{* *}$ \\
\hline Social support & & & & & & & .206 & .266 & $5.218^{* * *}$ & .099 & .128 & $3.085^{* *}$ \\
\hline Family function & & & & & & & & & & .636 & .616 & $13.786^{* * *}$ \\
\hline$F$ & & $3.152^{*}$ & & & $11.177^{* * *}$ & & & $14.004^{*}$ & & & $41.109^{\star}$ & \\
\hline$R^{2}$ & & .057 & & & .200 & & & .264 & & & .543 & \\
\hline F change & & $3.152^{*}$ & & & $56.014^{* * *}$ & & & $27.233^{*}$ & & & $190.062^{*}$ & \\
\hline$R^{2}$ change & & .057 & & & .143 & & & .064 & & & .279 & \\
\hline
\end{tabular}

Note. $N=322$.

${ }^{*} p<.05 .{ }^{* *} p<.01 .{ }^{* * *} p<.001$. 
적으로 유의미한 모형으로 관찰되었고 $(F=41.109, p<.001)$, 이혼가족 청소년이 경험하고 있는 비양육부모와의 관계 변 량 중 $54.3 \%$ 를 설명하고 있는 것으로 나타났다 $\left(R^{2}=.543\right)$. 최 종 모형인 4단계 모형에 새롭게 투입된 가족기능은 3단계 모 형에 투입된 인구사회학적 특성, 부모이혼지각, 사회적지지 의 영향력을 통제한 상태에서도 비양육부모와의 관계 변량 중 27.9\%를 통계적으로 유의미한 수준에서 설명하고 있었다 $\left(F\right.$ change $=190.062, p<.001, R^{2}$ change $\left.=.279\right) .4$ 단계에 투입 된 변수들 중에서는 통계적으로 유의미한 영향력을 보이지 않 았던 성별, 연령, 종교유무, 한부모가족 형성 기간과 3 단계까 지 유의미한 영향력을 보였던 경제수준과 형제 유무는 비양육 부모와의 관계에 유의미한 영향력을 갖지 못하는 것으로 나 타났다. 반면 부모이혼지각 $(t=3.041, p<.01)$, 사회적지지 $(t=$ $3.085, p<.01)$ 는 유의미한 영향력을 나타냈으며, 4단계에 투 입된 가족기능 $(t=13.786, p<.001)$ 역시 이혼가족의 청소년이 비양육부모와 맺고 있는 관계에 유의미한 영향력을 갖고 있었 다. 이는 부모이혼에 대한 문제성 신념이 낮고 긍정적으로 지 각할수록, 교사나 또래로부터의 사회적지지의 수준이 높을수 록, 현재 동거하고 있는 가족과의 원활한 상호작용을 통해 긍 정적인 기능을 형성할수록 비양육부모와의 긍정적인 관계를 형성할 수 있음을 의미한다. 비양육부모와의 관계에 유의미한 영향력을 갖는 요인들 중에서는 가족기능 $(\beta=.616)$ 이 가장 큰 영향력을 갖고 있었고, 부모이혼지각 $(\beta=.128)$, 사회적지지 $(\beta=.128)$ 의 순으로 상대적으로 큰 영향력을 갖고 있었다.

\section{논의 및 결론}

지속적으로 높은 수준을 유지하고 있는 이혼율과 상당수의 이 혼가족에 미성년 자녀가 있는 상황 하에서 이혼가족 청소년들 이 경험하는 어려움을 완화하고 이들의 성장을 지원할 수 있 는 중요한 요인으로 제시되고 있는 비양육부모와의 관계에 영 향을 주는 주요 요인을 살펴보았다. 본 연구에서는 특히 비양 육부모와의 관계에 영향을 주는 주요 요인으로 부모이혼지각, 사회적지지, 가족기능을 중심으로 각 변수의 영향력을 살펴보 았으며, 이를 근거로 한 논의의 결과는 다음과 같다.

첫 번째로 이혼가족 청소년들의 비양육부모와의 관계를 비 롯하여 부모이혼지각, 가족기능, 사회적지지의 특성을 살펴본 결과 비양육부모와의 관계 수준은 보통보다 약간 높은 수준 을 보이는 것으로 나타났다. 또한 비양육부모와의 관계 수준 에 영향을 주는 요인으로 제시한 부모이혼지각, 가족기능, 사
회적지지 역시 보통보다 약간 높은 수준을 보이고 있었다. 각 변수들과 비양육부모와의 관계가 보이고 있는 상호 관련성을 살펴본 결과에서는 부모이혼지각, 가족기능, 사회적지지 모두 정적인 관련성을 갖는 것으로 나타났다.

두 번째로 이혼가족 청소년의 비양육부모와의 관계에 영향 을 주는 주요 요인들을 살펴본 결과 부모이혼지각, 사회적지 지, 가족기능은 모두 유의미한 영향력을 갖는 것으로 나타났 다. 부모의 이혼 이후 함께 생활하는 가족과의 상호작용을 통 해 얻게 되는 가족기능은 비양육부모와의 관계에 유의미한 영 향력을 갖고 있는 것으로 나타났으며, 가족기능이 비양육부모 와의 관계에 영향을 준다는 선행연구(S. Y. Lee, 2002; Saphir \& Chaffee, 2002; Son, 2013)의 결과를 지지하는 것으로 볼 수 있 다. 비양육부모와의 관계에 대한 가족기능의 영향은 이혼가족 청소년이 가장 밀접하게 상호작용하는 체계이며, 이혼가족 청 소년의 긍정적.부정적 행동 형성의 가장 큰 영향력을 갖는 체 계라는 점을 반영한 것이다(Falci, 2006; H. Y. Kang \& Lazarevic, 2013; Risch, Jodl, \& Eccles. 2004). 또한 이혼가족 청소년에게 영향을 주는 두 개의 가족 체계 중 하나인 현재 생활하는 가족 과의 상호작용이 또 다른 가족체계인 비양육부모와의 관계에 영향을 주는 것으로 볼 수 있다. 부모의 이혼을 통해 경험하게 되는 부모이혼지각과 학교에서의 교사와 또래의 사회적지지 역시 비양육부모와의 관계에 유의미한 영향력을 갖고 있었으 며, 이는 부모이혼지각이 비양육부모와의 관계에 영향을 준다 는 선행연구(Joo \& Cho, 2004; Kurdek, 2002)와 사회적지지가 비양육부모와의 관계에 영향을 준다는 선행연구(Jang \& Lee, 2011; K. E. Lee \& Ju, 2005)의 결과를 지지하는 것으로 볼 수 있다. 부모이혼지각은 비양육부모에 대한 기본적인 태도를 형 성하는 인지적인 측면에 영향을 주는 특성이라는 점을 반영한 결과이다. 또한 부모 이혼 이후 이혼으로 인해 변화된 상황에 대한 수용을 통해 새롭게 변화된 관계에 적응하게 된다는 점 에서 부모이혼에 대해 문제성 인식이 낮을수록 비양육부모와 의 관계에 긍정적으로 영향을 주게 된다. 청소년기 가장 많은 시간을 보내게 되는 학교라는 공간에서 주요하게 관계를 맺게 되는 교사와 또래 체계는 청소년기의 행동 형성에 중요하게 영향을 주게 된다. 이는 비양육부모와의 관계에 가족과 함께 청소년기 중요한 영향을 주는 체계로써 학교 체계에서의 관계 가 갖는 중요성을 보여준다. 이혼가족 청소년의 비양육부모와 의 관계에 대한 부모이혼지각, 사회적지지, 가족기능의 상대 적인 영향력에서는 가족기능이 상대적으로 가장 높은 영향력 을 갖는 것으로 나타났고, 부모이혼지각, 사회적지지의 순으 로 높은 영향력을 갖는 것으로 나타나 이들에 대한 개입 및 관 
련 서비스의 제공 필요성을 확인할 수 있었다.

이혼가족 청소년의 비양육부모와의 관계에 대한 다른 영향 요인들을 통제한 상태에서 가족기능은 유의미한 독자적인 영 향력을 갖고 있는 것으로 나타났다. 이를 모형별로 살펴보면 인구사회학적 특성을 통제한 상태에서 부모이혼지각, 인구사 회학적 특성과 부모이혼지각을 통제한 상태에서 사회적지지, 인구사회학적 특성과 부모이혼지각, 사회적지지의 영향력을 통제한 상태에서도 가족기능은 각각 독자적인 설명력을 갖는 것으로 나타났다. 이는 이혼가족 청소년에게 영향을 주는 다 양한 체계 중 현재 함께 생활하는 가족과의 상호작용이 갖는 의미를 다시 확인할 수 있는 결과로 볼 수 있다. 연구모형의 변 화 중 3 단계까지 비양육부모와의 관계에 유의미한 영향력을 갖고 있던 경제수준과 형제유무가 가족기능이 투입됨으로 인 해 영향력을 갖지 않게 되는 것은 비양육부모와의 관계에 있 어 가족이 가진 경제수준이나 형제유무로 인한 구조적인 측 면 보다는 현재 동거하는 가족간의 상호작용인 기능적인 측면 이 중요함을 보여주는 것이라 할 수 있다. 특히 이혼가족에게 상대적으로 열악한 수준을 보이는 것으로 제시되고 있는 경제 수준으로 인해 나타나는 비양육부모와의 관계의 제한들을 현 재 함께 생활하는 가족들과의 상호작용인 가족기능에 대한 개 입을 통해 완화할 수 있음을 보여주는 것이라 할 수 있다. 또한 가족의 변화에 따라 지속적으로 논의되어 온 자녀에 대한 가 족기능과 가족구조의 영향력 중 가족이 갖는 구조적 특성에 비해 가족의 기능적 특성이 더 큰 영향력을 갖고 있음을 확인 한 연구들과 같은 맥락을 형성하는 것으로 볼 수 있다(Jeong, 2011).

이러한 연구결과를 바탕으로 이혼가족 청소년들의 비양육 부모와의 관계를 긍정적으로 증진하기 위해서는 이혼가족 청 소년에게 가장 큰 영향을 주는 체계이기도 하며, 비양육부모 와의 관계에 가장 영향력이 큰 요인이기도 한 현재 함께 생활 하는 가족과의 상호작용인 가족기능의 증진을 통한 개입이 필 요하다. 이혼 결정 과정과 이혼 이후 변화된 가족 체계는 부모 의 역할 과중과 사회· 경제적인 지위의 하락, 사회적지지체계 의 축소, 심리적 위축 등의 현상이 복합적으로 나타나면서 가 족기능의 왜곡을 가져올 가능성을 높인다(Y. L. Kim, 2014). 따 라서 이혼으로 이루어진 한부모가족을 대상으로 한 소득지원 정책을 포함하여 방과후 보호 제도의 활성화와 같은 사회적 서비스의 확대가 필요하다. 이러한 과정에는 변화된 가족 구 조와 기능에 대한 수용과 적응을 지원하기 위한 교육이 포함 되어져야 하며, 가족 구성원 상호간의 개방적인 태도 형성과 의사결정을 위한 원활한 의사소통, 상호간 지지 체계를 구축
하도록 원조함으로써 가족기능을 향상시키기 위한 노력이 수 반되어야 한다. 이를 위해 여성가족부와 관계부처 합동으로 3 차 건강가정기본계획 하에서 추진되고 있는 건강가정지원센 터를 중심으로 한 이혼가족에 대한 서비스 체계 구축과 강화 는 이혼가족의 가족기능을 향상시키는 중요한 역할을 수행할 수 있다. 이혼 가족의 특성에 따른 가족교육, 부모교육 및 상담 기능 강화 특히 이혼가족 청소년의 양육자를 대상으로 한 변 화된 자녀 양육 상황에 따른 양육방식에 대한 교육, 가족관계 증진을 위한 상담프로그램의 활성화는 이혼가족 청소년이 현 재 함께 생활하는 가족과 기능적인 상호작용을 하도록 함과 동시에 비양육부모와의 관계를 긍정적으로 향상시키는데 도 움을 주게 될 것이다(Jun, 2012; Y. K. Kim \& Lee, 2011). 이를 위한 가족교육과 가족상담 전문가 양성은 이혼가족의 가족기 능을 향상시키기 위한 서비스의 체계적 구축과 전문성 강화에 초석이 될 것이다. 전문가 양성 과정에 가족 및 이혼조정 실천 의 표준 모델 보급(Folberg, Milne, \& Salem, 2004)과 같은 개입 방법을 학습할 수 있는 기회를 제공하여야 하며, 장기적으로 는 우리나라의 실정에 맞는 개입 모델에 대한 개발의 진행이 요구된다.

또한 부모의 이혼을 경험하면서 청소년들이 갖게 되는 부 정적인 부모이혼지각에 대한 개입이 필요함을 알 수 있다. 아 동기의 경우 자기비난이나 유기불안 현상이 높고, 청소년의 경우에는 부모에 대한 비난이나 또래놀림 현상이 높은 수준을 보이는 것과 같이 부모의 이혼에 대해 갖게 되는 문제성 인식 은 연령단계에 따른 차이를 보인다(Wallerstein, 2000). 따라서 부모이혼지각에 대한 개입에 있어서 청소년을 대상으로 하는 경우에는 이혼부모에 대한 비난과 또래와의 관계에서 나타날 수 있는 위축을 문제를 완화할 수 있는 서비스가 요구된다. 부 모이혼지각에 대한 문제성 인식의 형성은 부모의 이혼 결정과 그 이후의 과정에서 자녀와 관련된 상황을 충분히 공유하는 못하는 제한된 의사소통과 관련이 깊은 것으로 제시되고 있 다(Emery, 2004). 따라서 부모의 이혼 과정에서 이혼과 관련된 상황들이 자녀들과 충분히 공유되어질 수 있도록 하는 교육 과 지도가 필요하다. 이를 위해 이혼 전후 상담, 이혼조정시의 상담 서비스 과정에서 자녀들이 충분히 참여할 수 있는 방안 의 모색과 가족의 변화로 인한 이상적 가족의 상실과 이로 인 한 부정적 감정을 표현할 수 있는 기회가 제공되어지는 협력 적 이혼 모델(Fagerstrom, 1997)과 같은 개입방법의 도입이 필 요하다.

이혼가족 청소년의 비양육부모와의 관계 향상을 위해서는 학교를 중심으로 한 사회적지지 체계의 강화가 필요하다. 학 
교에서 가장 많은 시간을 보내며 가장 많은 상호작용을 하는 청년기의 특성을 고려하여 볼 때 학교를 중심으로 한 개입은 반드시 필요하다. 부모의 이혼으로 인해 심리적 - 관계적 어려 움을 경험할 가능성이 높은 청소년들을 대상으로 교사 및 또 래와 긍정적인 관계를 유지할 수 있도록 지원하는 프로그램이 운영되어야 할 것이다. 교사와 학생들을 대상으로 하는 이혼 가족을 비롯한 다양한 가족에 대한 교육이 필요하다. 또한 같 은 어려움을 경험하는 청소년들을 대상으로 하는 학교 내 또 는 외부에서 진행될 수 있는 자조모임 활동 역시 이혼가족 청 소년들의 지지체계를 강화하는 효과를 가져올 수 있을 것이다 (K. J. Park, Choi, \& Han. 2009).

본 연구는 이혼가족 청소년의 성장에 중요한 영향을 주는 체계로 인식되어 왔지만 객관적인 실태 파악과 관련 변인에 대한 연구가 제한된 상황에서 비양육부모와의 관계에 영향을 주는 주요 요인을 검토하였다는 점에서 의의를 갖는다. 특히 국내에서 탐색적이고 제한적으로 수행되어져 온 비양육부모 와의 관계를 청소년에게 영향을 주는 다양한 체계로써 개인의 지각, 학교를 중심으로 한 상호작용, 현재 함께 생활하는 가족 과의 상호작용을 고려하여 부모이혼지각, 사회적지지, 가족기 능의 영향력을 객관적으로 검증하고, 특히 다른 변인들의 영 향력을 통제한 상태에서 가족기능이 갖는 독자적인 설명력의 확인을 통해 가족기능의 중요성을 확인하였다는 점에서 의의 를 갖는다. 하지만 연구대상자의 선정 과정에서 확률적인 표 집방법이 아닌 청소년상담기관, 중·고등학교, 사회복지기관 을 통해 선정된 대상자들로부터 수집된 자료를 통해 연구가 수행되어 그 결과를 전체 이혼가족 청소년들에게 일반함에 있 어 제한을 갖는다. 이러한 연구의 한계점은 향후 여성가족부 나 청소년정책연구원과 같은 행정기관이나 국가 연구 기관에 서 수행되어지는 전국단위의 후속 연구를 통해 극복될 수 있 다. 또한 이혼가족 청소년들이 경험하는 비양육부모와의 관계 에 대한 질적 연구방법을 활용한 심층적인 분석과 부모 이혼 결정부터의 한부모가족 형성 이후까지의 과정에 대한 변화시 기 및 단계별 자료 수집과 분석을 통해 각 단계의 변화에 대한 면밀한 검토와 이에 따른 필요한 개입방법에 대한 모색이 이 루어져야 할 것이다.

\section{Acknowledgements}

This study was supported by the National Research Foundation of Korea Grant funded by the Korean Government (NRF-
2013S1A5A8026005).

\section{Conflict of Interest}

No potential conflict of interest relevant to this article was reported.

\section{References}

Amato, P. R. (2000). The consequences of divorce for adults and children. Journal of Marriage and the Family, 62(4), 12691287.

Amato, P. R., \& Cheadle, A. (2005). The long reach of divorce: Divorce and child well being across three generations. Journal of Marriage and the Family, 67(1), 191-206.

Bae, M. L., \& Lee. K. M. (2006). Attachment and narcissistic personality of early adolescence: A comparison of only and sibling. Korean Journal of Family Welfare, 11(1), 113-130.

Carothers, S. S., Borkowski, J. G., Lefevre, J. B., \& Whitman, T. L. (2005). Religiosity and the socio-emotional adjustment of adolescent mothers and their children. Journal of Family Psychology, 19(2), 263-275.

Cho, Y. S., \& Lee, Y. S. (2004). The effect of social competence enhancement program on self-efficacy and interpersonal relation ability of middle school students from single-parent families. The Korean Journal of East West Mind Science, 7(2), $85-105$.

Choi, J. K. (2013). Adolescents' experience of living with divorced father: Giorgi's phenomenological approach. The Korean Journal of Youth Studies, 20(12), 73-99.

Chung, H. S. (1993). Post-divorce adjustment: The experience of the school children and adolescents. The Korean Journal of Child Studies, 14(1), 59-75.

Demaray, M. K., Malecki, C. K., Davidson, L. M., Hodgson, K. K., \& Rebus, P. J. (2005). The relationship between social support and student adjustment: A longitudinal analysis. Psychology in the Schools, 42(7), 691-706.

Depner, C., \& Bray, J. (1993). Nonresidential parenting: New vistas for family living. New York: Guilford Publication.

Emery, R. E. (1999). Postdivorce family life for children. In R. Thompson \& P. Amato (Eds.), The post divorce family: Children, parenting, and society. Thousand Oaks, CA: Sage.

Emery, R. E. (2004). The truth about children and divorce: Dealing with the emotions so you and your children can thrive. Viking/ Penguin: New York.

Epstein, N. B., Baldwin, L. M., \& Bishop, D. S. (1983). The McMaster Family Assessment Device. Journal of Marital 
and Family Therapy, 9(2), 171-180.

Fagerstrom, K. (1997). Divorce and family mediation: Models, techniques, and applications. Orinda, CA: Brookwood.

Falci, C. (2006). Family structure, closeness to residential and nonresidential parents, and psychological distress in early and middle adolescence. The Sociological Quarterly, 47(1), 123-146.

Felner, R. D. (2006). Poverty in children and adolescence. In S. Coldstein \& R. B. Brooksw (Eds.), Handbook of Resilience in Children. New York, NY: Springer.

Folberg, J., Milne, A. L., \& Salem, P. (2004). Divorce: A problem to be solved, not a battle to be fought. New York: Guilford Publication.

Haggerty, R., Sherrod, L., Garmezy, N., \& Rutter, M. (1996). Stress, Risk and Resilience in Children and Adolescents. New York: Cambridge University Press.

Hetherington, E. M. (2003). Social support and the adjustment of children in divorce and remarried families. Childhood, 10(2), 217-236.

Hong, S. H. (2004). The mediating effects of family income and parenting: The effect of parental divorce on psycho-social adjustment of adolescent children. Journal of the Korean Society of Child Welfare, 17, 151-177.

Jang, D. H., \& Lee, K. E. (2011). The experience of resilience in children of divorce. Journal of Future Oriented Youth Society, $8(4), 49-74$.

Jeong, S. H. (2011). Influences of single-parent family and parental conflict on children's mental health. Korean Journal of Social Welfare Studies, 42(4), 165-186.

Joo, S. H., \& Cho, S. W. (2004). Impact of conflict and nurturing factors for the divorced parents on the behavioral adaptation of their children. Korean Journal of Social Welfare, 56(4), 215237.

Ju, S. H. (2003). Children's beliefs on parental divorce and children adjustment problem on parent's post-divorce. Korean Journal of Family Social Work, 12, 182-213.

Jun, M. H. (2002). A study of the formulation process of coparenting types after divorce. Journal of Korean Family Therapy, 10(1), 51-73.

Jun, M. H. (2012). A study on the program for parenting education after separation and divorce. Korean Journal of Clinical Social Work, 9(1), 63-79.

Kang, E. S., \& Yi, H. Y. (2013). A preliminary survey for the parental perception of their adolescent children's emotional and behavioral adjustment during the consensual divorce. The Journal of Korea Single Parent Family, 6(1), 49-65.

Kang, H. Y., \& Lazarevic, V. (2013). Exploring adolescent-parent relationships in Asian American immigrant families: An ecological perspective. Child Studies in Asia-Pacific Contexts, 3(2), 105-122. doi:10.5723/csdc.2013.3.2.105
Kim, E. J., \& Baek, H. J. (2007). The effect of parental divorce on children's self-esteem: Based on the effects of family structure, economical deficiency and parenting. Family and Culture, 19(3), 79-103.

Kim, H. K., \& Chang, H. A. (2015). Effects of cognitivebehavioral group program for children of divorce on depression, self-esteem, and perception of divorce. The Korea Journal of Youth Counseling, 23(2), 423-445.

Kim, H. S. (2005). Post-divorce adjustment of children: Correlates of psychological resilience and the influence of Korean traditional family culture. The Journal of Education, 25(1), 277-302.

Kim, K. W., \& Moon, S. K. (2005). An analysis of ecological variables affecting parent-adolescent child relationship. The Korea Journal of Youth Counseling, 13(1), 71-84.

Kim, S. J. (2008). An investigation of divorce education program for divorcing parents in the USA-implications for the developing of divorce education program in Korea. Theology and Ministry, 30, 327-355.

Kim, Y. H., \& Han, G, H. (2006). Divorced noncustodial fathers' and mothers' contact and relationship satisfaction with children. Family and Environment Research, 44(8), 23-32.

Kim, Y. K., \& Lee, D. M. (2011). Effectiveness of solution-focused brief therapy for adolescents of divorced families. The Korean Journal of Youth Studies, 18(3), 49-81.

Kim, Y. L. (2014). Changes in social risk structure and family risk in Korea. Family and Culture, 26(2), 151-188.

Kurdek, L. A. (2002). A 1-year follow-up study of children's divorce adjustment, custodial mothers' divorce adjustment, and postdivorce parenting. Journal of Applied Developmental Psychology, 9(3), 315-328.

Kurdek, L. A., \& Berg, B. (1987). Children's beliefs about parental divorce scale: Psychometric characteristics and concurrent validity. Journal of Consulting and Clinical Psychology, 55(5), 712-718.

Lange, A., Blonk, R., \& Wiers, R. (1998). The parent-child interaction questionnaire, PACHIQ. Clinical Psychology and Psychotherapy, 5(3), 187-198.

Lange, A., Evers, A., Jansen, H., \& Dolan, C. (2002). The parentchild interaction questionnaire-revised. Family Process, 41(4), 709-722.

Lee, K. E., \& Ju, S. H. (2005). Factors influencing the adaptation of children in post-divorce stage: Related to patterns of child rearing. Journal of the Korean Society of Child Welfare, 20, 35-66.

Lee, S. Y. (2002). Study of adjustment of adolescents from divorced families. Study of Adjustment of Adolescents from Divorced Families, 10, 37-65.

Lee, S. Y. (2009). The effects of child counseling during divorce procedures. Asian Journal of Child Welfare and Development, 7(2), 35-49. 
Malecki, C. K., \& Elliott, S. N. (1999). Adolescents' ratings of perceived social support and its importance: Validation of the student social support scale. Psychology in the Schools, 36(6), 473-483.

Masten, A. S., \& Coastworth, J. D. (1998). The developments of competence in favorable and unfavorable environments: Lessons from research on successful children. American Psychologist, 53(2), 205-220.

Moon, H. J. (2007). A study for changed aspects after currency crisis in Korean society. Journal of International Area Studies, 11(1), 83-102.

Park, J. A., \& Yoo, S. K. (2003). Relationship of resilience, spiritual well-being and parental attachment. Korean Journal of Counseling and Psychotherapy, 15(4). 765-778.

Park, K. J., Choi, H. Y., \& Han, J. A. (2009). Children of divorced families. Korean Journal of Child Studies, 30(6), 323-336.

Park, Y. Y., \& Kim, K. E. (2005). Relationships between emotional competence and social adjustment among Korean children and adolescents. Korean Journal of Human Ecology, 14(1), $15-25$.

Risch, S. C., Jodl, K. M., \& Eccles, J. S. (2004). Role of the fatheradolescen relationship in shaping adolescents' attitudes toward divorce. Journal of Marriage and Family, 66(1), 46-58.

Saphir, M. N., \& Chaffee, S. H. (2002). Adolescent' contributions to family communication patterns. Human Communication Research, 28(1), 86-108.

Son, S. H. (2013). Divorced mothers' experiences of noncustodial fathers' involvement with their children and co-parenting relationships. Family and Environment Research, 51(4), 439454.

Statistics Korea. (2014). Marriage and Divorce Statistics in 2013. Daejeon, Korea: Author.

Wallerstein, J. S., Kelly, J. B., \& Blakelee, S. (2000). The unexpected legacy of divorce. New York: Hyperion.

Yoo, H. J. (2005). A case study about relationships between the non-custodial parents and the children \& coparenting after divorce. Korean Journal of Family Social Work, 15, 185-217.

Yoon, M. S., Lee, M. S., Kim, N. H., \& Jeong, H. S. (2012). A study on the mediating effects of self-esteem on the relationship between loss and depression among children in divorced families. Korean Journal of Family Social Work, 35, 73-104.

\section{ORCID}

Cho, Sung Hui http://orcid.org/0000-0002-1657-5373

Received March 24, 2016

Revision received April 27, 2016

Accepted April 27, 2016 